\title{
ANTIBIORÉSISTANCE ET « UNE SEULE SANTÉ » EN PRATIQUE : LE POINT DE VUE DU BACTERIOLOGISTE
}

\author{
ANTIMICROBIAL RESISTANCE AND ONE HEALTH IN PRACTICE: \\ THE BACTERIOLOGIST'S POINT OF VIEW
}

Par Mélanie PIMENTA'1, Yohann LACOTTE², Marie-Cécile PLOY ${ }^{3}$

(Note soumise le 7 octobre 2021, acceptée le 24 octobre 2021).

Mots clés : antibiorésistance, une seule santé, bactériologiste

Key words: antibioresistance, One Health, microbiologist

\section{INTRODUCTION}

La pandémie mondiale de Covid-19 nous a rappelé les dangers liés aux maladies infectieuses dont les zoonoses. Elle nous invite à repenser notre vision de la Santé et à la considérer comme un tout où hommes, animaux et environnements sont tous étroitement liés ; un concept ou paragdime baptisé "Une Seule Santé " ou encore "One Health" a été proposé en 2004. Celui-ci prône le recours à une approche collaborative et multidisciplinaire, basée sur le partage des savoirs et connaissances, pour faire face à plusieurs enjeux de Santé Publique majeurs émergents tels que la sécurité alimentaire (plusieurs ingestions d'aliments contaminés survenus en 2007 et 2008 ; Puschner \& Reimschuessel, 2011) et la lutte contre les zoonoses (grippe aviaire, Ebola, Zika, ...).

Parmi ces enjeux majeurs de Santé publique émergents, on retrouve également l'antibiorésistance, une pandémie silencieuse qui tue, chaque année, plus de 30000 personnes en Europe (Cassini et al. 2019). Les bactéries ne connaissent pas de frontières, peuvent se propager entre différents écosystèmes et s'échanger des résistances via des éléments génétiques mobiles. Pour espérer freiner la dissémination des résistances entre hommes, animaux et écosystèmes environnementaux, seule une approche holistique "One Health " impliquant des professionnels de différents secteurs et les citoyens, peut fonctionner. Ainsi, ces 20 dernières années, l'antibiorésistance a fédéré de nombreux acteurs autour d'enjeux communs One Health à différentes échelles et selon diverses stratégies (Lacotte \& Ploy, 2021). Dans cette lutte contre l'antibiorésistance, le bactériologiste joue un rôle important en analysant, de manière indifférenciée, des bactéries provenant de l'homme, de l'animal et de l'environnement. Il se place donc à l'interface du continuum One Health et permet de faire le lien entre professionnels des 3 secteurs. Ainsi, par ses connaissances, ses recherches et son action comme acteur de santé publique, le bactériologiste va pouvoir contribuer de bien des manières à la lutte contre l'antibiorésistance.

1- Doctorante, Université Limoges, INSERM, CHU Limoges, UMR 1092, 2 rue du Docteur Marcland, 87025 Limoges

2- Ingénieur, Université Limoges, INSERM, CHU Limoges, UMR 1092, 2 rue du Docteur Marcland, 87025 Limoges

3- Professeure, Université Limoges, INSERM, CHU Limoges, UMR 1092, 2 rue du Docteur Marcland, 87025 Limoges. Courriel : marie-cecile.ploy@unilim.fr 


\section{LE RÔLE DU BACTÉRIOLOGISTE DANS L'IDEN- TIFICATION DES DÉTERMINANTS DE LA RÉSISTANCE ET DE LEUR DISSÉMINATION}

Le bactériologiste joue un rôle majeur dans la caractérisation des résistances et de leur dissémination. Quotidiennement, le bactériologiste clinique, dans son activité d'analyse, isole et caractérise des bactéries résistantes, issues d'échantillons humains ou animaux, selon des techniques et référentiels standardisés (EUCAST, https://www.eucast.org/) tout en gardant certaines spécificités propres au monde humain ou animal via des référentiels dédiés. Ces isolats résistants serviront alors aux bactériologistes, plus fondamentaux, pour l'étude et la caractérisation des supports de la résistance.

L'essor du séquençage haut débit et des techniques multi-omiques permet aujourd'hui de caractériser rapidement et facilement les supports génétiques de la résistance. A l'heure du tout-numérique, les données générées par les bactériologistes alimentent de plus en plus souvent des bases de données informatiques aussi bien généralistes (i.e CARD, https://card. mcmaster.ca/ ; EMBL-EBI, https://www.ebi.ac.uk ; NDARO, https://www.ncbi.nlm.nih.gov/ ; ResFinder 4.1, https://www. genomicepidemiology.org) que dédiées à des résistances (Beta-Lactamase Data Base, http://bldb.eu) ou à des vecteurs de résistances particuliers tels que les intégrons (INTEGRALL, http://integrall.bio.ua.pt/), les plasmides (ACLAME, http://aclame.ulb.ac.be/) ou les séquences d'insertion (ISfinder, https://isfinder.biotoul.fr/). Autant d'outils aujourd'hui indispensables aux chercheurs et à la compréhension des mécanismes de la résistance.

Au-delà de la simple caractérisation des supports de la résistance, le bactériologiste contribue également à mieux comprendre comment ces gènes disséminent. Les analyses de clonalités permettent par exemple de retracer le parcours d'un germe ou de résistances entre différents réservoirs (Sirichokchatchawan et al. 2021). En couplant les données génomiques du bactériologiste à des approches bio-informatiques, il devient ainsi possible de retracer l'histoire évolutive de certains clones bactériens, plasmides et autres supports de résistance. Une récente analyse bio-informatique sur 10000 plasmides de référence a par exemple montré que $60 \%$ d'entre eux sont capables de dépasser la barrière de l'espèce et $10 \%$ la barrière de l'ordre, montrant bien l'importance des éléments génétiques mobiles dans la dissémination de la résistance entre espèces et réservoirs (Redondo-Salvo et al. 2020).

Enfin, le bactériologiste contribue également à limiter la dissémination des résistances en fournissant aux cliniciens de précieuses informations sur le phénotype de résistance de bactéries pathogènes. Le cas échéant, ces informations serviront aux prescripteurs pour modifier leurs antibiothérapies et privilégier des antibiothérapies ciblées à base d'antibiotiques à spectre réduit ou étroit moins susceptibles de (co-)sélectionner des résistances. Il est ainsi un acteur clé dans les pratiques de bon usage des antibiotiques.

\section{LE RÔLE DU BACTÉRIOLOGISTE DANS LE SUIVI ÉPIDÉMIOLOGIQUE DE LA RÉSISTANCE}

Le travail du bactériologiste va servir au suivi épidémiologique de la résistance à la fois (i) localement, lors d'épisodes épidémiques en établissement de santé ou dans un élevage, mais aussi (ii) nationalement en contribuant aux différentes missions de surveillance de la résistance en établissement de santé (SPARES, http://www.cpias-grand- est.fr/), dans la communauté (PRIMO, https://antibioresistance.fr/) ou chez les animaux (RESAPATH, https://resapath.anses.fr/). Cette surveillance existe aussi à l'échelle Européenne via des réseaux de laboratoires multinationaux. On peut notamment citer, le réseau Européen de surveillance chez l'homme, EARS-net, coordonné par l'ECDC (European Centre for Disease prevention and Control), (https://www.ecdc.europa.eu/), et bientôt son pendant chez l'animal, EARS-Vet (https://eu-jamrai.eu/help- build-ears-vet/) (Mader et al. 2021). Cette contribution du bactériologiste au suivi de l'épidémiologie est cruciale pour prévenir et contrôler efficacement la diffusion des déterminants de la résistance aussi bien localement que nationalement.

Le bactériologiste a également un rôle à jouer dans la détermination des indicateurs de surveillance les plus pertinents pour assurer le suivi épidémiologique de la résistance. De par sa place centrale à l'interface des 3 secteurs, Le bactériologiste participe à la définition des indicateurs (bactéries, marqueurs de résistance, ...) permettant de relier la surveillance humaine, animale et environnementale (par exemple les souches de Escherichia coli BLSE dans le protocole Tricycle de l'OMS ; OMS, 2021). Le bactériologiste a également un rôle à jouer dans la surveillance environnementale en définissant les indicateurs et marqueurs de résistance les plus pertinents pour assurer un suivi longitudinal de la résistance dans ce grand réservoir des résistances. Parmi ces indicateurs pertinents, on peut citer l'exemple des intégrons, structures génétiques vectrices de résistance, dont l'utilisation comme indicateur permet de refléter l'abondance totale des gènes de résistance aux antibiotiques, aux biocides ou encore aux métaux lourds, dans différents environnements (Stalder et al. 2012 ; Zheng et al. 2020).

\section{LE RÔLE DU BACTÉRIOLOGISTE DANS LA FORMATION ET LA VULGARISATION}

De par sa connaissance approfondie de la génétique bactérienne, le bactériologiste a un rôle important à jouer dans la formation et la vulgarisation. Au centre du continuum One Health, le bactériologiste est en mesure de faire le lien entre professionnels des trois secteurs. Il est donc un acteur privilégié pour former les professionnels du et au One Health. Il est aussi bien capable d'assurer un support technique sur la réalisation d'un prélèvement, que de fournir des indications sur un pathogène ou une résistance. Il a également un rôle à jouer dans la formation aux bonnes pratiques de prélèvement et de surveillance afin de 
rendre interopérables les données collectées par les professionnels des 3 secteurs.

La vulgarisation est également une des clés pour lutter contre l'antibiorésistance. En informant le grand public sur la diversité des bactéries et des résistances, sur leur capacité à disséminer entre les différents réservoirs et sur l'impact des antibiotiques sur cette dissémination, le bactériologiste est un acteur privilégié pour sensibiliser à l'antibiorésistance et au One Health. Les bactériologistes se sont d'ailleurs plutôt bien saisis de cette mission en France avec plusieurs réseaux de formation et/ou d'information impliquant des bactériologistes en santé humaine (AntibioEst, https://www.ant ibioest.org/ ; MATIS, https://www.preventioninfection.fr/), vétérinaire (R2A2, https://www6.inrae.fr/r2a2) ou environnementale (GRAIE, https://asso.graie.org/portail/), tous engagés dans des actions de sensibilisation à l'antibiorésistance et/ou au One Health.

\section{LE RÔLE DU BACTÉRIOLOGISTE DANS LA STRUCTURATION DU ONE HEALTH}

Le bactériologiste a également un rôle à jouer dans la structuration du One Health en particulier dans le champ de la recherche. Confrontés aux isolats (multi-)résistants, les bactériologistes sont en mesure de déterminer les pathogènes et résistances les plus problématiques. Ils participent à la définition des pathogènes prioritaires établies par l'OMS ; une liste qui façonne la recherche internationale en définissant des cibles de recherche prioritaires (OMS, 2017). Les bactériologistes sont aussi impliqués dans la définition des besoins en recherche autour de l'antibiorésistance, que ce soit à l'échelle française (plan prioritaire de recherche antibiorésistance, https://i3m.aviesan. fr/index.php?pagendx=927) ou européenne (JPIAMR - https://www.jpiamr.eu/, One Health EJP - https://on ehealthejp.eu/).
Au-delà du simple champ de la recherche, le bactériologiste possède un rôle structurant de par sa position privilégiée au centre du One Health. Ici encore, les bactériologistes se sont plutôt bien saisis de cette mission en France et se lancent dans des initiatives structurantes ambitieuses telles que les récents projets ABRomics-PF et PROMISE (https://ppr-antibioresistance.inserm.fr/), visant respectivement à créer une plateforme numérique sur la résistance antimicrobienne pour stocker, intégrer, analyser et partager des données multi-omiques et un méta-réseau professionnel One Health autour de l'antibiorésistance. A l'échelle européenne, des bactériologistes ont été investis à différents niveaux dans l'action conjointe Européenne de lutte contre l'antibiorésistance et les infections associées aux soins, EU. JAMRAI (https://eu-jamrai.eu/) ; une action conjointe permettant de faire le lien entre les trois secteurs et de promouvoir des actions concrètes One Health pour lutter efficacement contre l'antibiorésistance à l'échelle européenne.

\section{CONCLUSION}

Au centre du continuum One Health, les bactériologistes, qu'ils soient de formation en médecine vétérinaire ou médecine humaine ou spécialistes d'écologie bactérienne ou de génétique bactérienne, jouent un rôle primordial dans la caractérisation des résistances et de leurs modes de dissémination mais aussi dans leur suivi épidémiologique. Véritable lien entre les professionnels du One Health, ils ont aussi un rôle à jouer dans la formation des professionnels, dans les actions de sensibilisation et la structuration du One Health en aidant par exemple à l'identification de priorités de recherche. Les bactériologistes se sont saisis de ces enjeux en France et l'on peut se réjouir de les voir de plus en plus souvent engagés dans des initiatives ambitieuses de formation, de sensibilisation et de structuration.

\section{BIBLIOGRAPHIE}

- Cassini A, Högberg LD, Plachouras D, Quattrocchi A, Hoxha A, Simonsen GS et al. Attributable deaths and disability-adjusted life-years caused by infections with antibiotic-resistant bacteria in the EU and the European Economic Area in 2015: a population-level modelling analysis. The Lancet Infectious Diseases. 2019 ; 19, 56-66.

- Lacotte Y \& Ploy M-C. Antibiorésistance à l'échelle d' "Une Seule Santé " : stratégies, enjeux, opportunités. Bulletin de l'Académie Vétérinaire de France. 2021 ; sous presse.

- Mader R, Damborg P, Amat J-P, Bengtsson B, Bourély C, Broens EM et al., Building the European Antimicrobial Resistance Surveillance network in veterinary medicine (EARS-Vet). Euro Surveill. 2021; 26:pii=2001359.

- OMS, Organisation Mondiale de la Santé. Global priority list of antibiotic-resistant bacteria to guide research, discovery, and development of new antibiotics. 2017. Disponible à : https://www.who.int/medicines/publ ications/WHO-PPL-Short_Su mmary_25Feb-ET_NM_WHO.pdf (consulté le 04.10.2021)

- OMS, Organisation Mondiale de la Santé. WHO integrated global surveillance on ESBL-producing E. coli using a "One Health" approach: Implementation and opportunities. 2021. Disponible à : https://www.who.int/ publications/i/item/who-integ rated-global-surveillance-on-esbl-produc ing-e.-coli-using-a-one-health-approach (consulté le 04.10.2021)

- Puschner B \& Reimschuessel R. Toxicosis caused by melamine and cyanuric acid in dogs and cats: uncovering the mystery and subsequent global implications. Clin Lab Med. 2011;31:181-199.

- Redondo-Salvo S, Fernández-López R, Ruiz R, Vielva L, de Toro M, Rocha
EPC et al. Pathways for horizontal gene transfer in bacteria revealed by a global map of their plasmids. Nature Communications. 2020; 11, 3602. - Sirichokchatchawan W, Apiwatsiri P, Pupa P, Saenkankam I, Khine NO, Lekagul A, et al. Reducing the risk of transmission of critical antimicrobial resistance determinants from contaminated pork products to humans in South-East Asia. Front Microbiol. 2021; 12, 689015-689015.

- Stalder T, Barraud O, Casellas M, Dagot C, Ploy MC. Integron involvement in environmental spread of antibiotic resistance. Front Microbiol. 2012; 3, 119. - Zheng W, Huyan J, Tian Z, Zhang Y, Wen X. Clinical class 1 integron-integrase gene. A promising indicator to monitor the abundance and elimination of antibiotic resistance genes in an urban wastewater treatment plant. Environment International. 2020; 135, 105372. 